\title{
Design of Switches \& Sockets Testers based on Microcontroller
}

\author{
Abdelrasoul Jabar Alzubaidi 1, Yousif Taha Yousif Elamin \\ 1 Electronics Dept. - Engineering College - Sudan University of science and Technology \\ 2 Electronics Dept. - Faculty of Engineering \& Technology - University of Gezira.
}

\begin{abstract}
It's important to maintain the quality of the electrical and electronic devices (electrical household switches, sockets-outlet, .etc), since they pose danger to the users. These devices affect directly the national economy.

Most of the international and national organizations including the Sudanese standard and metrology organization have paid so much concern to the electrical devices quality through stabling a lot of standard specifications to control its quality.

Regarding the electrical household switches and sockets-outlet, all the standard specifications which have been established are required.

The difficulties of simulating the normal operating test manually for the electrical household switches, and sockets come from the needs of perfectly performing the following tests:

- Pregnancy current.

- Electrical endurance.

For electrical household switches (up to 15A, 250V) according to Sudanese standard SDS (3926/2007), and socket-outlet (up to12.5A,230V) according to Sudanese standard SDS (4829/2011), which required the on/off times for electrical switches to be approximately equal.

So in this paper two separate devices are subjected to tests. The first one is for the electrical household switches and the second is for the sockets-outlet.
\end{abstract}

\section{INTRODUCTION}

Electricity is so much part of modern living that we can often take it for granted. It is a powerful and versatile energy but can be dangerous if it is not used properly. Serious accidents with electricity in the home are rare but each year there is a small number of fatalities and serious injuries. Most of the accidents that occur are due either carelessness or to a lack of awareness of some basic rules that should always be observed when using electricity.

Electrical and, electronic equipments become obsessed many industrialized countries and consuming countries, Sudan is among them. Sudan import a lot of products, including appliances electrical and electronic equipments, which has become indispensable in the daily life of consumers in urban and rural areas, including switches and sockets of electrical household. Most of the equipments and household appliances are imported from various sources. This leads to a lot of machinery and electrical equipment are poor quality and weak posing a danger to the consumer, national economy and the electrical /electronic devices that connect to them.

Many organizations and international cooperation bodies and institutions, regional and national issue has made many standard specifications in all fields in order to work on quality control of products, including:

ISO: International Standards Organization.

IEC: International Electrotechnical Commission.

SSMO: Sudanese Standard and Metrology Organization.

SASO: Saudi Arabian Standard Organization.

The Standard specifications (IEC-60669) is considered (switches for household and similar fixed -electrical installation) and taken by Sudanese standard specification (3926/2007) and other standard specifications issued by the ISO. IEC reference for many of the member states of these organizations, including the Sudan and these countries are modifying these specifications in line with their economic, environmental and climate.

This paper is built on the Sudanese standard specifications (SDS) (3926/2007) for electrical household switches. The (SDS) includes many tests. Parts of them are normal operation (pregnancy current test and electrical endurance test).

To perform the pregnancy current test, chose three switches. These three chosen switches must be able to carry the rated current for half an hour, and the potential different between each contact surfaces must not exceed 0.075 volt, also the temperature in any part of the switch should not exceed $15^{\circ} \mathrm{c}$.

Then the switch must pass successfully the same test again after the first 4000 operation cycle of the electrical endurance test, the switch plate must be tied in its place during the test. [1]In the electrical endurance test, use the same three switches that are used in the pregnancy current test. They must be able to carry 15000 complete operations (ON/OFF), these cycles include the first 4000 cycles of the Pregnancy current test. You can take out 
the switch plate often the first 4000 cycles for easy tracing the test. The time between ON/OFF must be approximately equal. The switches must be valid to use after the end of the test. [1]

This design is also fit to work on test:

- Electrical endurance of socket-outlet.

Sudanese specification SDS (4829/2011) reference (IEC 60884, SASO 443) concerning the plugs and sockets outlets for household and similar general use $230 \mathrm{~V} / \mathrm{up}$ to $12.5 \mathrm{~A}$, provides the following:

-Electrical endurance of socket - outlet;

Socket outlets should be capable of making and breaking the rated current 15,000 times in a substantially noninductive AC circuit.

- Electrical endurance of switch, socket - outlet;

Switch, socket-outlet should be able to connect and disconnect the rated current 15,000 completed cycles $(\mathrm{ON} / \mathrm{OFF})$, in a non-inductive $\mathrm{AC}$ circuit, at a rate not exceeding six complete cycles per minute at regular intervals.

\section{APPROACH}

This design is for conducting quality test of household electrical switches and electrical sockets for the normal operation and electrical endurance. It is described as follows:

- Electronic design: This section is especially important for practical design based on embedded system and programming.

- Mechanical design: Common mechanical loads can be classified with respect to their inertia and to the torque speed characteristic.

\section{SYSTEM COMPONENTS AND DESIGN}

The microcontroller Pic 16F877A is used in the design. C language is used for programming the microcontroller. The most important hardware components are mention bellow;

- Microcontroller Pic16F877A.

- Cristal oscillator.

- LED diode.

- Hall Effect sensor.

- Hex Schmitt trigger.

- Seven Darlington Arrays.

- Hex Schmitt inverter.

- Optocoupler.

- LM 555 timer.

- Push buttons switches.

- Emergency switch.

- Capacitors.

- Coil.

- Resistances.

- Transformer (220 -24 VAC).

- Rectifier.

- Regulators.

- LCD monitors.

- Air compressor.

- Directional control valves.

- Service unit.

- Flow control valves.

- Cylinders.

- Common base.

- Fittings.

- Air pipes.

The block diagram for the design is shown in figure (1). 


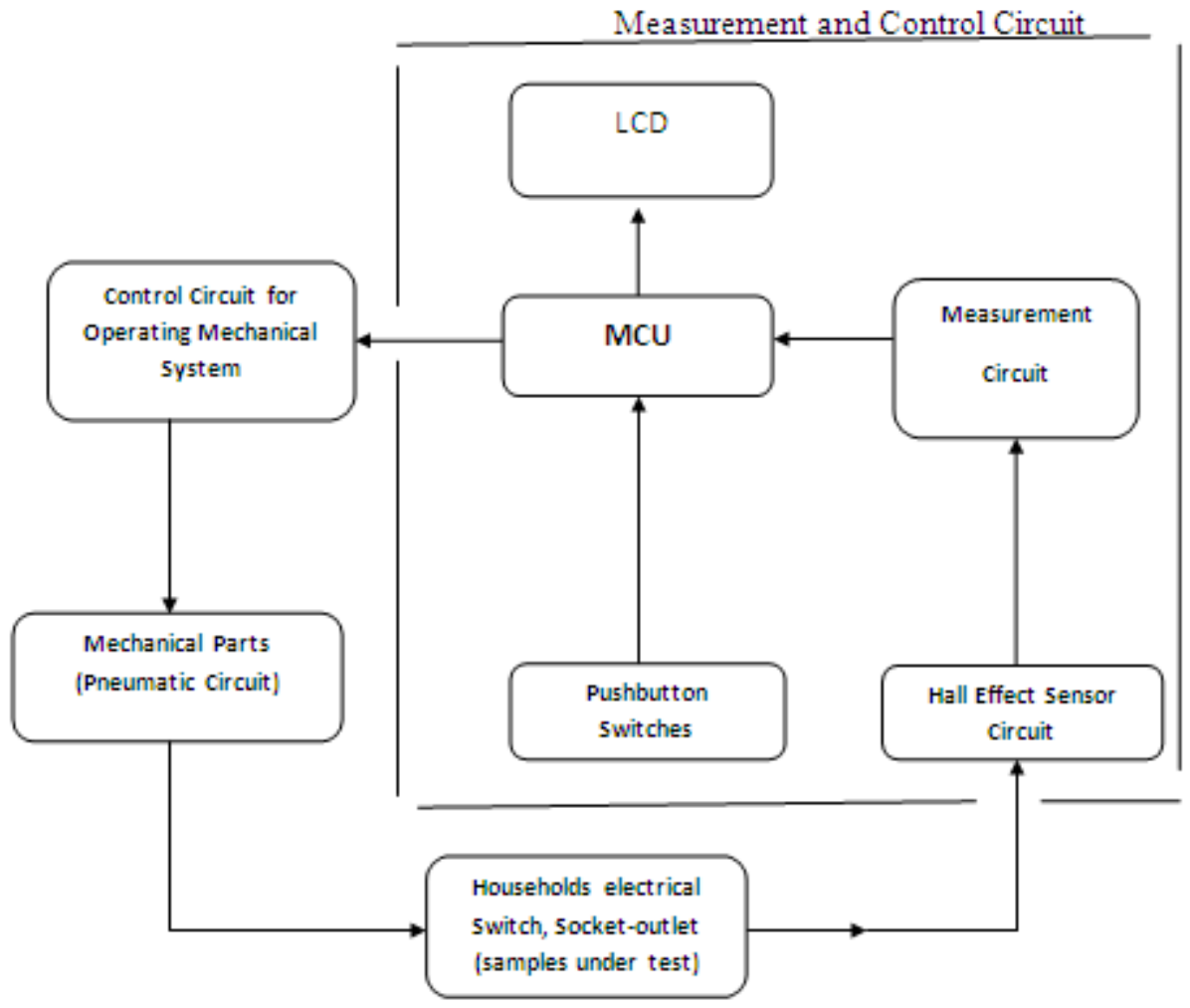

Figure (1) Devices Block Diagram

IV. ALGORITHM

The proposed computer algorithm includes a strict sequence of steps for the operation of the tester The algorithm is:

The program in this design will be prepared by $\mathrm{C}$ language and used a complier (mikro $\mathrm{C}$ programmer). The following algorithms for switches and sockets devices test:

- Algorithm for Switches devices test:

Start

Phase one:

--- Perform 4000 times ON operations.

- If any problem occurs activate FAIL subroutine.

- End the test procedure.

- If no problem occurs go to phase two of the test.

Phase two:

--- Perform 11000 times ON operations.

- If any problem occurs activate FAIL subroutine.

- End the test procedure.

- If no problem occurs activate PASS subroutine.

- End the test procedure.

End

- Algorithm for sockets device test:

Start

Phase one:

--- Perform 15000 times ON operations.

- If any problem occurs activate FAIL subroutine.

- End the test procedure

- If no problem occurs activate PASS subroutine.

- End the test procedure.

End 
V.

RESULTS

Following are the results for implementing the proposed design.

1. Electrical household switch sample test of electrical endurance:

- FAIL percentage of the electrical household switch samples test $=90 \%$

2. Electrical household switch sample test of the pregnancy current test:

- FAIL percentage of the electrical household switch samples test $=20 \%$

3. Electrical socket- outlet sample test of the electrical endurance:

- FAIL percentage of the electrical household switch samples test $=100 \%$

\section{CONCLUSION}

It is essential to conduct the quality control tests for different samples of the household switches and sockets. The pass and fail samples for the electrical endurance and the pregnancy current tests are stored in a data base as a reference for these samples.

\section{REFERANCES}

[1] Sudanese standard specification SDS (4829/2011).

[2] 2-Embedded Systems -Architecture, Programming and Design, second Edition, Raj Kamal, 2008.

[3] The Pic Microcontroller, Book 1, Microchip, Nebojsa Matic, May15, 2000.

[4] Microcontrollers [Theory and Application], Ajayv Deshmukh, by Tata McGrow -Hill, 2005.

[5] Pic Microcontrollers [know It All], Lucio Di Jasio, Tim Wilmshurst, Dolan Ibrahim, John Morton, Martin Bates, Jack Smith, D.W. Smith, and Chuck Hellebuyck, ISBN: 978-0-7506-8615-0, 2008, Elsevier Inc. .

[6] Interfacing Pic Microcontroller - Embedded Design by Interactive Simulation, Martin Bates, Edition-1, 2006.

[7] Measurement and Instrumentation Systems, W. Bolton, Edition-1, 1996.

[8] Pic 16F877A Data Sheet.

[9] Introduction to Microcontrollers, Courses 182.064 \& 182.074, Vienna University of Technology, Institute of Computer Engineering, Embedded Computing Systems Group ,February 26, 2007, Version 1.4, G*unther Gridling, Bettina Weiss.. 